\title{
Patrones de Cualidad Vocal en Psicoterapia: Desarrollo y Estudio de Confiabilidad de un Sistema de Codificación
}

\section{Vocal Quality Patterns in Psychotherapy: Coding System Development and Reliability Analysis}

\author{
Alemka Tomicic \\ Pontificia Universidad Católica de Chile \\ Luis D. Chacón \\ Pontificia Universidad Católica de Chile
}

\author{
Claudio Martínez \\ Universidad Diego Portales
}

\author{
Marco Guzmán \\ Universidad de Chile
}

\author{
Alejandro Reinoso \\ Pontificia Universidad Católica de Chile
}

\begin{abstract}
Se expone el sistema de codificación de Patrones de Cualidad Vocal (PCV) y su estudio de confiabilidad. El objetivo fue crear un sistema que permitiese identificar y codificar PVC por medio de una valoración auditiva entrenada. El diseño fue exploratorio-descriptivo y de enfoque mixto, con una muestra intencionada de 12 episodios de cambio extraídos de 3 psicoterapias. Mediante técnicas cualitativas fueron descritos 6 PCV: Reporte, Conectado, Afirmativo, Reflexión, EmocionalExpresivo y Emocional-Contenido. Posteriormente, por medio del coeficiente kappa de Cohen se analizó el grado de concordancia entre jueces independientes en la codificación de los PCV, sobre un total de 516 segmentos, correspondientes a 457 turnos de habla. El sistema mostró una alta confiabilidad inter-jueces y, entre sus ventajas, la posibilidad de analizar procesos psicoterapéuticos, sin importar su orientación teórica o enfoque, y su aplicabilidad al estudio de los PCV empleados por terapeutas y pacientes.
\end{abstract}

Palabras clave: mediciones acústicas, patrones de cualidad vocal, proceso psicoterapéutico

The coding system Vocal Quality Patterns (VQP) and a reliability study are presented. The aim was to create a system to identify and codify VQP using a trained hearing assessment. The design was descriptive-exploratory, employing a mixed approach, with a convenience sample of 12 episodes of change extracted from 3 psychotherapies. Qualitative techniques were used to describe 6 VQP: Report, Connected, Affirmative, Reflection, Emotional-Expressive and Emotional-Restrained. Subsequently, by Cohen's kappa coefficient the degree of agreement between independent judges, for the coding of the VQP for a total of 516 segments, corresponding to 457 talking turns, was examined. The system showed high inter-rater reliability and, among its advantages, it presents the possibility of analyzing psychotherapeutic processes, regardless of theoretical orientation or approach, and its applicability to the study of VQP used by therapists and patients.

Keywords: acoustic measures, vocal quality patterns, psychotherapy process

Alemka Tomicic, Programa de Doctorado en Psicología, Pontificia Universidad Católica de Chile, Santiago, Chile; Claudio Martínez Guzmán, Facultad de Psicología, Universidad Diego Portales, Santiago, Chile; Luis Diego Chacón, Programa de Magíster en Psicología, Pontificia Universidad Católica de Chile, Santiago, Chile; Marco Guzmán, Escuela de Fonoaudiología, Universidad de Chile, Santiago, Chile; Alejandro Reinoso, Centro de Salud Mental San Joaquín, Pontificia Universidad Católica de Chile, Santiago, Chile.

La correspondencia relativa a este artículo debe ser dirigida a Alemka Tomicic P., Programa de Doctorado en Psicología, Pontificia Universidad Católica de Chile, Avda. Vicuña Mackenna 4860, Macul, Santiago, Chile. E-mail: atomicic@uc.cl 
Destacados investigadores y teóricos en el campo de la psicoterapia han reconocido que la voz del terapeuta y el paciente constituye un aspecto no verbal fundamental para la comprensión de la interacción terapéutica (Beebe, Jaffe, Lachmann, Feldstein, Crown \& Jasnow, 2000; Bucci, 2007; Campanelli, 2008, Junio; Erickson, 1980; Osatuke et al., 2005; Russell, 1993; Wiseman \& Rice, 1989). Sin embargo, a pesar de la relevancia asignada a la voz en el diálogo psicoterapéutico, en la actualidad hay escasez de instrumentos que posibiliten el estudio científico de la cualidad de la voz en psicoterapia (Tomicic, Martínez, Altimir, Bauer \& Reinoso, 2009).

Dado lo anterior, para este estudio se consideró la creación de un sistema que permitiese la identificación y codificación de patrones de cualidad vocal (PCV) por medio de una valoración auditiva entrenada. Además, se desarrolló el Manual de Entrenamiento y Procedimiento para la Codificación de Patrones de Cualidad Vocal (Tomicic, Chacón et al., 2009) con el propósito de hacer accesible el sistema, tanto a investigadores como a psicoterapeutas interesados en el comportamiento vocal de los participantes en la interacción psicoterapéutica. ${ }^{1}$

\section{La Relevancia de la Voz en el Diálogo Psicoterapéutico}

De acuerdo con Andersen (1999), parámetros sonoros de la voz, como el ritmo, el tiempo, la resonancia, el control y el acento, resultan primordiales para la interpretación del habla. Señala que, en términos generales, la comunicación no verbal resulta significativa en la transmisión de información, siendo el principal vehículo para la comunicación a nivel relacional e interpersonal. Plantea que, entre los códigos contextuales no verbales, los vocales son los más importantes ya que estos refuerzan o modifican lo dicho verbalmente.

Por su parte, Campanelli, Iberni, Sarracino, Degni y Mariani (2007) han hecho referencia a las propiedades semánticas autónomas de la comunicación vocal no-verbal al identificar funciones de las claves vocales no verbales o paralingüísticas. En la interacción psicoterapéutica los parámetros sonoros del habla constituyen una de las principales fuentes de información sobre el sentido de los actos realizados en la comunicación entre terapeuta y paciente (Knoblauch, 2000, 2005). Se ha planteado que la cualidad de la voz del hablante influiría en el estado emocional de quien lo escucha (Bachorowski \& Orwen, 2008; Russel, Bachorowski \& FernandezDol, 2003), siendo posible, por ejemplo, que una voz que refleje el estado relajado y confiado del terapeuta pudiese calmar la voz agitada y las consecuentes emociones del paciente (Bady, 1985). Scherer y Bergmann (1990) encontraron en un estudio sobre comunicación vocal que en el proceso psicoterapéutico los participantes infieren y provocan emociones en el otro a través de los sonidos del habla. Knoblauch $(2000,2005)$ ha señalado que la cualidad vocal de paciente y terapeuta puede ser uno de los aspectos clave para la descripción de los procesos de cambio en psicoterapia. Señala que en la interacción psicoterapéutica se intercambiarían significados psicológicos a través del sonido de sus voces.

\section{El Estudio de la Voz en la Interacción Psicoterapéutica}

En las áreas de la fonética y la fonoaudiología se han hecho múltiples intentos por investigar y analizar las características vocales, siendo algunas de las principales el tono, la intensidad, el timbre, el ritmo y la flexibilidad (Dodero, Hortas \& Wilder, 2005; Le Huche \& Allali, 1993; Scivetti \& Garraza, 1996). Sin embargo, estos intentos han correspondido al área de la lingüística o de la salud para la adecuada producción de la voz.

En el campo de la psicoterapia son escasos los estudios empíricos sobre la voz y los que existen se han enfocado mayoritariamente en el análisis de la expresión vocal de las emociones (véase Bachorowski \& Owren, 1995, 2008, 2009; Moneta, Penna, Loyola, Buchheim \& Káchele, 2008; Scherer, 1982, 1986). Es decir, se han centrado en la investigación del habla centrada

\footnotetext{
Los interesados en poner en práctica el sistema de valoración e identificación auditiva de PCV que aquí se presenta pueden solicitar el entrenamiento y el manual contactándose con la primera autora.
} 
en el hablante - línea de estudio que asume que diferentes estados emocionales se asocian a diferentes elecciones léxicas, estilos de habla y fraseos- y se ha prestado menor atención a la investigación del habla centrada en la comunicación, línea de estudio que asume una modulación prosódica del habla en la interacción (Campbell, 2007).

En una investigación pionera centrada en la comunicación, Rice y Wagstaff (1967) desarrollaron un sistema de clasificación de patrones vocales para analizar la cualidad vocal de pacientes y para determinar el valor pronóstico de la presencia diferencial de estos PCV en el proceso de cambio psicoterapéutico. Distinguieron cuatro PCV en los pacientes - emocional, focalizado, externalizado y limitado- y establecieron relaciones entre la presencia de estos y la productividad de las sesiones evaluadas desde la perspectiva de los terapeutas (Rice \& Kerr, 1986; Rice \& Wagstaff, 1967). Posteriormente, Wiseman y Rice (1989) describieron seis PCV para los terapeutas y encontraron efectos significativos de la cualidad vocal del terapeuta sobre la cualidad vocal del paciente. Observaron que la cualidad vocal productiva del terapeuta podía predecir un cambio en el paciente hacia un patrón vocal enfocado, de conexión con la experiencia.

Más recientemente, Holtz (2004) desarrolló una investigación sobre la coordinación del ritmo vocal de terapeutas y pacientes en el curso de tres psicoterapias breves. Encontró que la interacción terapéutica se caracteriza por la interdependencia de dichos comportamientos vocales.

El presente trabajo recoge las ideas del estudio de Rice y Wagstaff (1967) tomando como punto de partida la clasificación de patrones vocales en pacientes desarrollada por estas autoras. El propósito fue recrear un sistema único de codificación de PCV para paciente y terapeuta, aplicable al diálogo psicoterapéutico más apropiado al contexto cultural chileno y, eventualmente, hispano-parlante. Los PCV fueron definidos como una combinación de parámetros vocales particulares en las alocuciones de los hablantes que, en la sonoridad del habla, impresiona a quien la escucha de una manera específica, independientemente del contenido de lo hablado (Tomicic, Chacón et al., 2009).

Con respecto a esto último, existe evidencia proveniente de estudios experimentales que apoya la hipótesis que plantea que la expresión vocal de emociones se caracteriza por elementos que son universalmente empleados por los hablantes y pueden ser decodificados con niveles de precisión que superan los esperados por el azar, independientemente del idioma de quien escucha (Scherer, Banse \& Wallbott, 2001; Thompson \& Balkwill, 2006). Sin embargo, también se ha reportado evidencia que subraya la ventaja comparativa del reconocimiento de emociones expresadas a través de la prosodia del habla por parte de oyentes que comparten el mismo idioma del hablante (Pell, Monetta, Paulmann \& Kotz, 2009). En este sentido, Pell et al. (2009) han argumentado que, si bien la habilidad de reconocer emociones a través de la expresión vocal es parcialmente independiente de la correspondencia idiomática de quien escucha e implica principios universales, también es cierto que esta habilidad es modulada por variables lingüísticas y culturales. Desde esta perspectiva, la consideración de estas variables en el desarrollo del presente sistema de codificación de PCV contribuye a la confiabilidad y validez ecológica en la aplicación del mismo.

\section{Método}

El diseño fue de tipo exploratorio-descriptivo, utilizando secuencialmente técnicas cualitativas y cuantitativas.

\section{Muestra}

Tanto para la elaboración del sistema de codificación de PCV como para la realización del estudio de confiabilidad, la unidad muestral considerada fue el episodio de cambio psicoterapéutico (Krause, De la Parra, Arístegui \& Strasser, 2006, 2008; Krause et al., 2007).

Para la realización del estudio de confiabilidad del sistema se empleó una muestra de 12 episodios de cambio provenientes de tres psicoterapias individuales de diferentes orientaciones 
teóricas ${ }^{2}$. De cada uno de estos tres procesos psicoterapéuticos se analizaron cuatro episodios de cambio, tomados de cuatro sesiones diferentes. Además, para examinar la aplicabilidad del sistema de codificación de PCV con pacientes y terapeutas de diferente sexo, se buscó que las tres psicoterapias seleccionadas estuviesen compuestas por duplas paciente-terapeuta que representaran distintas combinaciones de voz masculina y femenina dentro de las posibilidades existentes (véase la Tabla 1). No se contó con la combinación terapeuta mujer-paciente hombre, pues el registro de audio de la única psicoterapia disponible con estas características no cumplía con los requisitos mínimos para una codificación por medio de apreciación acústica entrenada.

Tabla 1

Muestra del Estudio

\begin{tabular}{cccc}
\hline $\begin{array}{c}\text { Proceso } \\
\text { psicoterapéutico }\end{array}$ & Sujetos & $\begin{array}{c}\text { Episodios de } \\
\text { cambio }\end{array}$ & $\begin{array}{c}\mathrm{N}^{\mathrm{o}} \text { de la } \\
\text { sesión }\end{array}$ \\
\hline Proceso I & 1 & 1 \\
Psicodinámico & Terapeuta Hombre & 2 & 3 \\
& Paciente Hombre & 3 & 5 \\
Proceso II & 4 & 5 \\
Psicodinámico & Terapeuta Hombre & 2 & 1 \\
& Paciente Mujer & 3 & 4 \\
Proceso III & & 4 & 21 \\
Cognitivo conductual & Terapeuta Mujer & 2 & 1 \\
& Paciente Mujer & 3 & 4 \\
Total & & 4 & 5 \\
& 3 terapeutas y 3 & 12 & 12 \\
\hline
\end{tabular}

\section{Procedimiento}

Las sesiones de terapia tuvieron lugar en una sala de espejo de visión unidireccional y fueron grabadas en audio y video para permitir análisis posteriores.

Los pacientes y sus terapeutas fueron extensivamente informados sobre los propósitos de las investigaciones antes del inicio de las terapias. Asimismo, los pacientes y sus terapeutas consintieron respecto del registro y recolección de datos durante todo el proceso de psicoterapia y firmaron consentimientos informados en los que se explicitó el resguardo de su anonimato y

2 Los registros de audio y transcripciones de las psicoterapias utilizados en este trabajo forman parte del acervo de de datos recolectados en tres proyectos de investigación financiados por Fondos de Investigación de la Vicerrectoría Adjunta de Investigación y Doctorado de la Pontificia Universidad Católica de Chile (VRAID N ${ }^{\circ}$ 1080136, 2007) y Fondos para el Desarrollo de Ciencia y Tecnología, Ministerio de Educación, Gobierno de Chile (FONDECYT N ${ }^{\circ}$ 1030482, 2003 y N 1060768, 2007). 
el uso exclusivo de los datos recolectados para propósitos de investigación. Todos estos procedimientos fueron certificados por el comité de ética de la Escuela de Psicología de la Pontificia Universidad Católica de Chile.

\section{Análisis de la Información}

La elaboración del sistema de codificación de PCV se desarrolló en tres pasos sucesivos: (a) identificación de parámetros vocales, (b) identificación y descripción de los PCV y (c) caracterización y validación del sistema de codificación de PCV.

Identificación de parámetros vocales. Para la identificación y el análisis de los parámetros vocales cuatro de los autores del artículo participaron de un entrenamiento con un fonoaudiólogo especialista en el reconocimiento de voces, con el objetivo de capacitar al equipo en el reconocimiento auditivo de parámetros de la voz. Habiendo delimitado parámetros vocales que podían ser reconocidos auditivamente, el equipo realizó una codificación preliminar de las voces de una dupla terapeuta mujer-paciente mujer, cuyo propósito fue seleccionar los parámetros vocales a emplear para caracterizar los PCV. Como resultado, el tono, la intensidad, la duración y el timbre (ver Tabla 2) fueron definidos como aquellos parámetros vocales que, mediante una apreciación auditiva entrenada, permitían caracterizar la cualidad vocal de paciente y terapeuta.

Tabla 2

Definición de los Parámetros Vocales

\begin{tabular}{|c|c|}
\hline $\begin{array}{c}\text { Parámetro } \\
\text { vocal }\end{array}$ & Definición \\
\hline Tono & $\begin{array}{l}\text { Corresponde, en términos de medición objetiva, a la frecuencia de las ondas sonoras y, en términos } \\
\text { de la percepción humana, a la evaluación de lo agudo o grave del sonido y a su carácter melódico. } \\
\text { Considera los subparámetros de Prosodia, Tono medio hablado, Producción del acento, Final de } \\
\text { frase. }\end{array}$ \\
\hline Intensidad & $\begin{array}{l}\text { Corresponde, en términos de medición objetiva, a los decibeles (unidad de comparación de magnitudes) } \\
\text { y, en términos de la percepción humana, a la evaluación de la energía o volumen de la voz. Considera } \\
\text { los subparámetros de Volumen medio y Variaciones-Dinámica. }\end{array}$ \\
\hline Duración & $\begin{array}{l}\text { Corresponde, en términos de medición objetiva, a los segundos y minutos de extensión del sonido } \\
\text { y, en términos de la percepción humana, a la evaluación de un sonido como más largo o más corto. } \\
\text { Considera los subparámetros de Ataque vocal, Velocidad del habla, Ritmo del habla y Pausas. }\end{array}$ \\
\hline Timbre & $\begin{array}{l}\text { Corresponde, en términos de medición objetiva, a la frecuencia y amplitud de las ondas sonoras } \\
\text { que determinan el tono fundamental y sus respectivos armónicos y, en términos de la percepción } \\
\text { humana, a la cualidad particular de la voz de determinada persona. Considera los subparámetros de } \\
\text { Flexibilidad y Color-Brillo. }\end{array}$ \\
\hline
\end{tabular}

Identificación y descripción de patrones de cualidad vocal. Se realizó un análisis fenomenológico (Álvarez-Gayou, 2004; Rodríguez, Gil \& García, 1996) con el que, por medio de la escucha entrenada, fueron identificados, descritos y nombrados seis PCV. Este análisis consistió en la descripción de los turnos de habla, en términos de la manera como su sonoridad impresionaba la escucha de los investigadores. Este primer análisis adscribió al principio general del enfoque fenomenológico de producir descripciones exhaustivas de la experiencia subjetiva ante un fenómeno dado, de modo de obtener una comprensión de la estructura esencial del fenómeno en sí (McLeod, 2001). Específicamente, se buscó describir la cualidad que le imprimían a los turnos de habla analizados diferentes características sonoras, independientemente del contenido de lo hablado. 
En esta etapa participaron cuatro miembros del equipo, quienes se reunieron en un primer momento a escuchar, sin instrucción alguna, extractos de diálogos psicoterapéuticos. Luego de dos sesiones siguiendo este esquema, se realizó un brain-storming, en el que cada uno nominó y describió las cualidades sonoras distinguidas en los extractos escuchados. Los seis PCV fueron nominados por consenso intersubjetivo. En las siguientes dos sesiones cada PCV nominado fue descrito exhaustivamente por el equipo de investigación, utilizando un método en el que se alternaba la escucha de los extractos de audio y la descripción de las impresiones que permitió distinguir cada PCV en su estructura fenomenológica. Este procedimiento se repitió hasta lograr saturación en las descripciones de cada uno de los PCV (Ponterotto, 2006).

Elaboración y validación del sistema de codificación de PCV. Una vez nominados y descritos los seis PCV, se realizó una primera codificación a un episodio de cambio, correspondiente a una sesión de terapia con un terapeuta hombre y una paciente mujer. Con esta codificación se buscó caracterizar cada PCV en términos de la percepción auditiva de parámetros vocales específicos definidos previamente. Cada PCV fue caracterizado con parámetros primarios y secundarios, con el objetivo de establecer claves sonoras para su identificación y diferenciación respecto de cada uno de los otros PCV.

Con el fin de asegurar la confiabilidad del sistema de codificación de PCV, posteriormente se realizó un estudio de confiabilidad en tres etapas relativamente superpuestas:

Etapa 1. Se desarrolló un manual para describir el sistema de codificación de PCV (Tomicic, Chacón et al., 2009), el cual muestra la organización del sistema, caracterizando cada uno de los seis PCV y describiendo los procedimientos para practicar la discriminación auditiva de los parámetros vocales y realizar la codificación empleando los PCV. Un aspecto importante de la exposición del sistema de codificación de PCV que se implementó en el manual fue la incorporación de ejemplos inventados en audio de cada uno de los parámetros vocales y PCV, empleando para ellos una voz masculina y una femenina, respectivamente, utilizando siempre la misma frase para los parámetros vocales:

¿Sabe? me quedé pensando en lo que me contó la sesión pasada sobre sus padres. Hábleme un poco más acerca de eso.

Y utilizando, también, la misma frase para los PCV:

... pero, es que es el reto que tengo... si yo renunciara a ese reto me quedaría sin nada o sea con nada, por eso mismo si es el reto que tengo y no tengo otra salida, como también se barajarán otras opciones, también las aceptaría, pero la verdad es que no las hago.

Estos ejemplos pueden ser escuchados con el fin de practicar la discriminación auditiva de los parámetros vocales y los PCV, o con el propósito de discernir la codificación de un PCV a través de la comparación del segmento de audio que es objeto de análisis.

Etapa 2. Siguiendo las indicaciones del manual, se llevó a cabo un ejercicio de codificación de PCV en dos episodios de cambio. Este fue realizado en conjunto por el equipo de investigación, con el propósito de consensuar los procedimientos de codificación antes de dar inicio al estudio de confiabilidad.

En esta etapa se determinó el turno de habla como la unidad de análisis de los PCV, se establecieron reglas para su segmentación y las condiciones mínimas que cada segmento debía cumplir para que la codificación de un PCV fuera posible (Tomicic, Chacón et al., 2009; ver Tabla 3 y Figura 1). 
Tabla 3

Definición de la Unidad de Análisis, Segmentación y Condiciones Para la Codificación de PCV

Unidad de análisis, segmentación y condiciones

\author{
Definición
}

Turno de habla

En términos de los registros de audio, los límites del turno de habla son definidos por el cambio de actor (paciente, terapeuta) que emite la vocalización.

Durante la codificación de PCV el turno de habla puede ser segmentado Segmentación del turno de habla en subunidades solo en el caso que en este se aprecien dos PCV distintos. Para poder segmentar el turno del habla, el nuevo PCV debe presentarse de manera evidente. De lo contrario, se privilegia la codificación del PCV predominante.

Para la codificación de los PCV se requiere como mínimo que la alocución tenga una duración de dos segundos. Esta duración mínima permite la percepción de los parámetros vocales principales para la confirmación de los PCV Reflexión, Conectado y Afirmativo. Para el caso del PCV Reporte la duración de la alocución deberá ser como mínimo de cinco segundos,

Condiciones mínimas para la segmentación y/o codificación de un PCV puesto que algunos de los parámetros principales (variaciones de la dinámica y ritmo) para la confirmación de este PCV requieren un tiempo mayor para su apreciación.

En el caso de que la alocución no cumpla con el tiempo mínimo de duración para ser codificada con el PCV hipotetizado (paso 3 del procedimiento de codificación) y no corresponda a una Pausa Llena o Solapamiento, se codifica como No codificable.

Asimismo, se establecieron cuatro pasos para el procedimiento de codificación de los PCV (Tomicic, Chacón et al., 2009):

1. Escuchar de una sola vez el episodio analizado, con el propósito de familiarizarse con el timbre de voz de los participantes.

2. Escuchar desde el inicio el episodio analizado, leyendo el texto turno por turno de habla, y realizar una segmentación preliminar, considerando cambios o quiebres en la cualidad vocal, dados por cambios en algún parámetro vocal (por ejemplo, cambios en la velocidad, el volumen o el ritmo).

3. Escuchar desde el inicio el episodio analizado, turno por turno de habla y segmento por segmento definido, y realizar una codificación preliminar, considerando la descripción fenomenológica de los PCV. En este paso la descripción fenomenológica de los PCV permite confirmar, descartar o precisar la segmentación realizada en el paso anterior.

4. Escuchar desde el inicio el episodio analizado, turno por turno de habla, y corroborar o descartar la presencia del PCV codificado en el paso 3, considerando la percepción auditiva de los parámetros de cualidad vocal principales y secundarios, dirimiendo en el caso de considerar más de un PCV por segmento. 


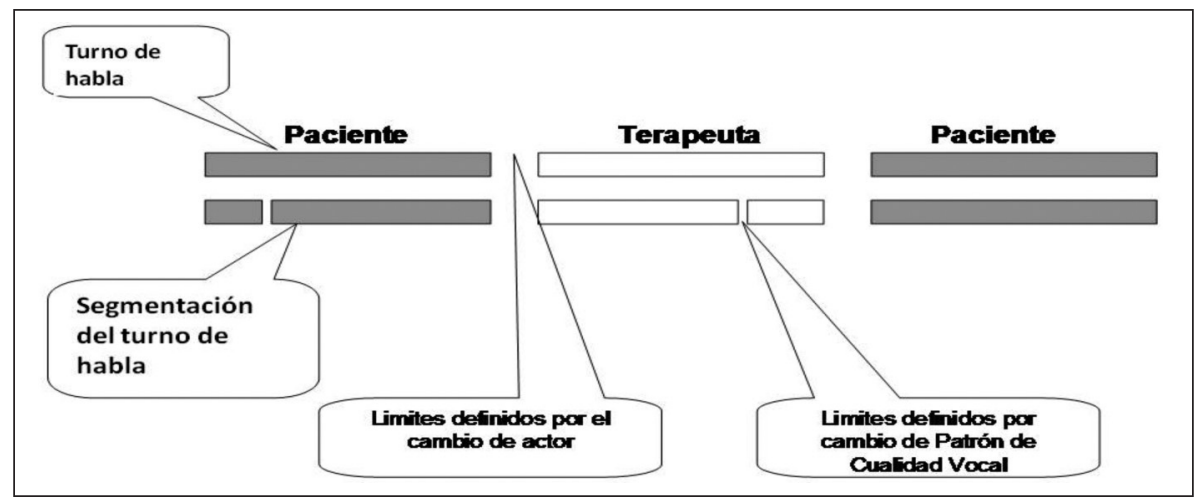

Figura 1. Unidad de análisis y segmentación del turno de habla para la codificación de PCV.

Etapa 3. Finalizado el ejercicio de codificación de los dos episodios de cambio, se realizó un estudio de confiabilidad para analizar las fuentes de desacuerdos y corregirlas en el manual. Este proceso fue realizado por dos miembros del equipo de investigación, empleando seis episodios de cambio extraídos de sesiones de una psicoterapia individual, extendiéndose el proceso hasta alcanzar un nivel de confiabilidad aceptable para el sistema de codificación de PCV (ver Tabla 4).

Tabla 4

Coeficientes Kappa del Estudio de Confiabilidad

\begin{tabular}{lccccc}
\hline $\begin{array}{c}\text { Intentos en } \\
\text { orden cronológico }\end{array}$ & Turnos de habla & $\begin{array}{c}\text { Segmentos o } \\
\text { unidades de } \\
\text { análisis }\end{array}$ & Kappa & Error & $p$ \\
\hline Episodio 1 & 14 & 22 & 0,48 & 0,12 & 0,05 \\
Episodio 2 & 25 & 36 & 0,45 & 0,08 & 0,05 \\
Episodio 3 & 23 & 30 & 0,39 & 0,09 & 0,05 \\
Episodio 4 & 30 & 38 & 0,65 & 0,09 & 0,05 \\
Episodio 5 & 19 & 22 & 0,82 & 0,12 & 0,05 \\
Episodio 6 & 31 & 36 & 0,89 & 0,06 & 0,05 \\
Total & 142 & 184 & & & \\
\hline
\end{tabular}

Nota. La muestra utilizada para el estudio de confiabilidad corresponde a seis episodios de cambio de sesiones de una psicoterapia individual terapeuta hombre-paciente hombre. 
Una vez logrado el nivel de confiabilidad aceptable para el sistema de codificación de PCV, se realizó el análisis de confiabilidad definitivo sobre la muestra de 12 episodios de cambio que se detallan en la Tabla 1. En este análisis participaron los mismos dos miembros del equipo de investigación que realizaron el primer estudio de confiabilidad ya descrito.

Los análisis de confiabilidad fueron realizados con el coeficiente kappa de Cohen, empleando los programas estadísticos SPSS 14.0 y EPIDAT 3.1 Este último es un programa para análisis epidemiológico de datos tabulados.

Se emplearon tres criterios para valorar los coeficientes kappa obtenidos en cada una de las codificaciones. En primer lugar, se consideró la significación estadística de cada kappa, de manera de concluir que los niveles de acuerdo obtenidos fuesen mayores a los que pudiesen ser alcanzados producto del azar. En segundo lugar, se empleó el criterio consensuado de valoración de los coeficientes kappa, en el que se plantea que un kappa mayor que 0,61 refleja un nivel de acuerdo aceptable y que un kappa mayor que 0,81 refleja un muy buen nivel de acuerdo (Landis \& Koch, 1977). En tercer lugar, dado que la evaluación de un valor kappa como aceptable o como muy bueno responde a un consenso de la comunidad científica, que no necesariamente se ajusta a las particularidades y diferentes exigencias de cada sistema de codificación (por ejemplo, número de categorías, diferencias en la probabilidad de ocurrencia de cada una de las categorías y nivel de precisión esperado, el que corresponde a probabilidad condicional de que un evento sea categorizado como $\mathrm{C} 1$ cuando en realidad corresponde a $\mathrm{C} 1$ [Bakeman, McArthur, Quera \& Robinson, 1997]), se empleó como criterio de valoración de los coeficientes kappa obtenidos el cálculo del valor kappa mínimo aceptable para el sistema de codificación de PCV. Para calcular este valor se empleó el software FalliObs (Bakeman et al., 1997) y se definieron las siguientes particularidades y exigencias al sistema de codificación de PCV: nueve categorías (seis PCV y tres categorías de exclusión), variabilidad moderada en la probabilidad simple de ocurrencia de las categorías y un nivel de $85 \%$ y $90 \%$ de precisión. Dadas estas especificaciones, se determinó que el valor kappa mínimo aceptable para el sistema de codificación de PCV es de 0,69 y 0,79, respectivamente.

\section{Resultados}

Los resultados se han organizado de la siguiente manera: en primer lugar, se presenta el sistema de codificación de PCV; en segundo lugar, se presentan los resultados del estudio de confiabilidad definitivo realizado al sistema de codificación de PCV y, finalmente, se presenta un ejemplo de la aplicación del sistema.

\section{Los Patrones de Cualidad Vocal}

En el actual sistema de codificación se establecieron y caracterizaron seis PCV. Estos fueron denominados: (a) Reporte, (b) Conectado, (c) Afirmativo, (d) Reflexión, (e) Emocional-Expresivo y (f) Emocional-Contenido. Además, para aquellas alocuciones en las cuales no se aplica la codificación de PCV se crearon las siguientes categorías: (g) Pausa Llena, (h) Solapamiento y (i) No Codificable (ver Tabla 5). 
Tabla 5

Caracterización de Patrones de Cualidad Vocal

\begin{tabular}{|c|c|}
\hline PCV & Caracterización \\
\hline Reporte & $\begin{array}{l}\text { Imprime al habla la cualidad de lo conocido, de desafección respecto de lo dicho y/o de } \\
\text { cierta distancia emocional. Suena como si el hablante estuviese reportando, contando } \\
\text { o indagando un contenido sin participación afectiva. En este patrón lo central es la } \\
\text { impresión en el oyente de un habla desafectada. }\end{array}$ \\
\hline Conectado & $\begin{array}{l}\text { Imprime al habla la cualidad de estar orientada hacia un otro y de estar siendo } \\
\text { elaborada en el mismo momento de su emisión. En este patrón lo central es la impresión } \\
\text { en el oyente de un habla elaborativa y orientada al otro. }\end{array}$ \\
\hline Afirmativo & $\begin{array}{l}\text { Imprime al habla la cualidad de certeza y convicción. Suena como si el hablante } \\
\text { estuviese aleccionando o instruyendo al oyente, o como si estuviese muy comprometido } \\
\text { o convencido de lo dicho. En este patrón lo central es la impresión en el oyente de un } \\
\text { habla segura e instructiva. }\end{array}$ \\
\hline Reflexión & $\begin{array}{l}\text { Imprime al habla la cualidad de estar orientada hacia uno mismo (el propio hablante). } \\
\text { Suena como si el hablante estuviese en conexión con su mundo interno o en un diálogo } \\
\text { consigo mismo. En este patrón lo central es la impresión en el oyente de un habla } \\
\text { ensimismada. }\end{array}$ \\
\hline $\begin{array}{l}\text { Emocional } \\
\text { expresivo }\end{array}$ & $\begin{array}{l}\text { Imprime la cualidad de afección y/o de que el habla está fuertemente cargada de } \\
\text { emocionalidad. Suena la emoción del hablante (alegría, rabia, tristeza, miedo, etc.). } \\
\text { En este patrón lo central es la impresión en el oyente de un habla emocionada, } \\
\text { independiente del tipo de emoción. }\end{array}$ \\
\hline $\begin{array}{l}\text { Emocional } \\
\text { contenido }\end{array}$ & $\begin{array}{l}\text { Imprime la cualidad de afección y/o de que el habla está fuertemente cargada de } \\
\text { emocionalidad. Sin embargo, en este caso no suena la emoción del hablante, sino que } \\
\text { al oyente lo impresiona un esfuerzo de contención de la emoción. En este patrón lo } \\
\text { central es la impresión de sofocamiento y control que evita el desborde emocional. }\end{array}$ \\
\hline
\end{tabular}

Categorías de exclusión para los PCV

Corresponde al caso del habla simultánea que, para efectos de la codificación del PCV,

Solapamiento impide la distinción de las características vocales de los participantes en un segmento o un turno de habla completo. Al codificar este fenómeno de la conversación se consigna el solapamiento para ambos actores.

Corresponde al caso de vocalizaciones breves y cuyo contenido es para-verbal ( $\mathrm{mmh}$, aha, ya). Suele ser una manera de asentir, comunicar atención, desacuerdo o el deseo Pausa llena de acabar la conversación. Su sentido depende principalmente del contexto y de ciertas características vocales de la enunciación; sin embargo, por su brevedad es muy difícil analizar en estas vocalizaciones los parámetros vocales que definen los PCV considerados.

Corresponde al caso de unidades de análisis que no cumplen con las características fenomenológicas y de parámetros de los PCV. También puede aplicarse en los casos en

No codificable que no se escucha adecuadamente el audio, por ruidos de contexto o por inadecuada pronunciación o articulación de alguno de los actores. No corresponde, a su vez, a una pausa llena ni a un solapamiento. 
Como se puede apreciar en la Tabla 5, cada uno de los PCV es descrito según la manera como estos impresionan a quien los escucha. Específicamente, se da cuenta de la cualidad que cada PCV imprime al habla independientemente del contenido de lo hablado. Así, por ejemplo, el PCV Reporte suena como un habla desafectada, como si el hablante estuviese reportando, contando o indagando un contenido sin participación afectiva. No obstante, este PCV es codificado independientemente de si el contenido de lo hablado coincide o no con un reporte, relato o pregunta e independientemente de que en este se empleen o no expresiones verbales emocionales.

Se buscó que todos los códigos que componen el Sistema de Codificación de PCV (seis PCV y tres categorías que excluyen la codificación de PCV) fuesen exhaustivos, es decir, todos los segmentos son susceptibles de ser codificados con alguna de las categorías del sistema y mutuamente excluyentes, aplicándose solo una categoría a cada segmento (Bakeman \& Quera, 1995). No obstante lo anterior, la descripción fenomenológica de cada uno de los PCV no siempre permite establecer distinciones absolutas entre cada uno de ellos. Tanto el PCV Reporte como el PCV Afirmativo imprimen al habla la cualidad de certeza: el primero, por el atributo de $l o$ conocido y el segundo, por el atributo de la convicción. Sin embargo, el aspecto diferenciador es que en el PCV Reporte al oyente lo impresiona un habla desafectada, mientras que en el PCV Afirmativo al oyente lo impresiona un habla comprometida con lo dicho. Otro ejemplo es el caso de los PCV Conectado y PCV Afirmativo. Ambos patrones imprimen al habla la cualidad del compromiso con lo dicho; sin embargo, el aspecto diferenciador es la impresión de certeza o incertidumbre en el oyente. En este sentido, el PCV Conectado imprime al habla un carácter elaborativo, mientras que el PCV Afirmativo le imprime seguridad. Más claras son las diferencias entre el PCV Conectado y el PCV Reflexivo. Mientras que el primero le imprime al habla la cualidad de estar orientada al otro, el segundo le imprime la cualidad de estar orientada al propio hablante. Sin embargo, en algunas ocasiones, sobre todo cuando resalta su carácter elaborativo, el PCV Conectado puede dar la impresión de un habla reflexiva. Finalmente, la distinción entre los PCV Emocional-Expresivo y PCV Emocional-Contenido resulta más sencilla en su descripción fenomenológica, pues aun cuando ambos patrones imprimen al habla la cualidad de estar cargada de intensa emocionalidad, reflejan dos polos opuestos. En el caso del PCV Emocional-Expresivo la emoción del hablante "suena" e incluso modula el aspecto verbal del habla. En cambio, en el PCV Emocional-Contenido la emoción es sofocada en el habla, impresionando al oyente el esfuerzo de control que realiza el hablante para evitar su desborde emocional.

Dadas las limitaciones en la distinción de los PCV que presenta la descripción fenomenológica por sí sola, cada uno fue descrito, adicionalmente, en términos de parámetros vocales. Se distinguió entre parámetros vocales principales y secundarios, según la importancia que estos tienen para diferenciar cada PCV. Los parámetros vocales principales de un PCV son aquellos que necesariamente deben presentarse para clasificar dicho patrón como tal. En cambio, los parámetros vocales secundarios son aquellos que contribuyen a identificar a un PCV determinado, pero sin ser necesaria su presencia para clasificarlo como tal.

Como se muestra en la Tabla 6, los PCV han sido caracterizados en términos de parámetros vocales que los identifican y al mismo tiempo los diferencian entre sí. Por ejemplo, el PCV Reporte puede ser caracterizado por la utilización de parámetros vocales principales asociados a la intensidad (volumen elevado y altas variaciones en la dinámica) y la duración (velocidad aumentada). En cambio, el PCV Afirmativo puede ser caracterizado por la utilización de parámetros vocales principales asociados al tono (acentuación dinámica y/o tónica y un final de frase en suspensión), la intensidad (dinámica sostenida y creciente) y la duración (inicio vocal duro). 
Tabla 6

Patrones de Cualidad Vocal y sus Parámetros Vocales Característicos

\begin{tabular}{|c|c|}
\hline Patrones de cualidad vocal & Parámetros vocales caraterísticos \\
\hline Reporte & $\begin{array}{l}\text { PRINCIPALES } \\
\text { Intensidad: volumen aumentado y variaciones altas } \\
\text { Duración: velocidad aumentada } \\
\text { SECUNDARIOS } \\
\text { Acento: dinámico-agógico-tónico } \\
\text { Final de frase: semi anticadencia-anticadencia } \\
\text { Inicio vocal: duro-suave-soplado } \\
\text { Ritmo: fluido } \\
\text { Pausas: cortas }\end{array}$ \\
\hline Conectado & $\begin{array}{l}\text { PRINCIPALES } \\
\text { Tono: acento dinámico-agógico } \\
\text { Final de frase: semi anticadencia-suspendido } \\
\text { Intensidad: volumen aumentado, dinámica sostenido-crescendo y variaciones } \\
\text { bajas } \\
\text { SECUNDARIOS } \\
\text { Inicio vocal: suave } \\
\text { Velocidad: aumentada-media } \\
\text { Ritmo: fluido con algunas disrupciones } \\
\text { Pausas: largas-medias }\end{array}$ \\
\hline Afirmativo & $\begin{array}{l}\text { PRINCIPALES } \\
\text { Tono: acento dinámico-tónico } \\
\text { Final de frase: suspendido } \\
\text { Intensidad: dinámica sostenida-crescendo } \\
\text { Duración: inicio vocal duro } \\
\text { SECUNDARIOS } \\
\text { Volumen: aumentado } \\
\text { Variaciones: bajas } \\
\text { Velocidad: aumentada } \\
\text { Ritmo: fluido } \\
\text { Pausas: cortas }\end{array}$ \\
\hline Reflexión & $\begin{array}{l}\text { PRINCIPALES } \\
\text { Tono: acento dinámico-agógico } \\
\text { Final de frase: semi cadencia-suspendido } \\
\text { Intensidad: volumen disminuido y variaciones bajas } \\
\text { Duración: velocidad disminuida } \\
\text { SECUNDARIOS } \\
\text { Inicio vocal: suave-soplado } \\
\text { Ritmo: fluido con algunas disrupciones } \\
\text { Pausas: largas-medias }\end{array}$ \\
\hline Emocional Expresivo & $\begin{array}{l}\text { PRINCIPALES } \\
\text { Intensidad: volumen aumentado y variaciones altas } \\
\text { Duración: velocidad aumentada } \\
\text { Pausas: cortas } \\
\text { SECUNDARIOS } \\
\text { Acento: dinámico-agógico-tónico } \\
\text { Final de frase: cadente, sostenido o anticadente }\end{array}$ \\
\hline $\begin{array}{l}\text { Emocional } \\
\text { Contenido }\end{array}$ & $\begin{array}{l}\text { PRINCIPALES } \\
\text { Duración: velocidad aumentada } \\
\text { Ritmo: no fluido } \\
\text { Pausas: largas } \\
\text { SECUNDARIOS } \\
\text { Acento: dinámico-agógico } \\
\text { Final de frase: suspendido-semi anticadencia } \\
\text { Volumen: disminuido } \\
\text { Inicio vocal: suave }\end{array}$ \\
\hline
\end{tabular}




\section{Estudio de Confiabilidad}

Se analizó el grado de concordancia entre jueces independientes para la codificación de seis PCV y tres categorías de codificación que excluyen los PCV. Como se aprecia en la Tabla 7, se realizó el análisis a un total de 516 segmentos, correspondientes a 457 turnos de habla de 12 episodios de cambio.

\section{Tabla 7}

Análisis de Confiabilidad de los PCV

\begin{tabular}{lcccccc}
\hline \multicolumn{1}{c}{$\begin{array}{c}\text { Patrones de cualidad vocal en } \\
\text { episodios de cambio }\end{array}$} & $\begin{array}{c}\text { Turnos } \\
\text { de } \\
\text { habla }\end{array}$ & $\begin{array}{c}\text { Segmentos o } \\
\text { unidades de } \\
\text { análisis }\end{array}$ & Kappa & Error & $p$ \\
\hline Dupla T. hombre - P. hombre & 76 & 93 & 0,71 & 0,05 & $<0,001$ \\
Dupla T. hombre - P. mujer & 206 & 222 & 0,84 & 0,02 & $<0,001$ \\
Dupla T. mujer - P. mujer & 175 & 201 & 0,83 & 0,02 & $<0,001$ \\
Total & 457 & 516 & 0,83 & 0,02 & $<0,001$ \\
\hline
\end{tabular}

Nota. $\mathrm{T}=$ terapeuta $\mathrm{P}=$ paciente.

Como se muestra en la Tabla 7, la codificación de PCV en episodios de cambio arrojó un $k=0,71, p<0,001,95 \%$ IC [0,60, 0,82] para el análisis de los episodios de la dupla terapeuta hombre-paciente hombre; un $k=0,84, p<0,001,95 \%$ IC $[0,79,0,90]$ para el análisis de episodios de la dupla terapeuta mujer-paciente mujer y un $k=0,83, p<0,001,95 \%$ IC $[0,79,0,90]$ para el análisis de los episodios de la dupla terapeuta hombre-paciente mujer.

Adicionalmente, los valores kappa obtenidos para el análisis de los episodios de cada dupla terapeuta-paciente fueron comparados entre ellos para examinar la presencia de diferencias estadísticamente significativas que pudiesen sugerir un efecto de la dupla en la confiabilidad del sistema. La prueba de homogeneidad de kappa, empleada para contrastar la hipótesis nula de que los coeficientes kappa comparados son iguales, arrojó que estos no presentan diferencias estadísticamente significativas $\chi^{2}(2, N=3)=4,89, p=0,086$. Además, fue posible obtener un kappa global, $k=0,83, p<0,001,95 \%$ IC $[0,79,0,86]$, correspondiente al conjunto de los episodios de cambio codificados con el sistema PCV.

Los coeficientes kappa obtenidos para cada dupla por separado son estadísticamente significativos $(p<0,001)$, lo que indica que los niveles de acuerdo son mayores a los que pudiesen ser obtenidos por el azar. Además, el kappa global supera significativamente el criterio consensuado para la valoración de kappa y el valor kappa mínimo aceptable propuesto previamente para el sistema de codificación PCV.

\section{Ilustración de la Aplicación del Sistema de Codificación de PCV}

A continuación se presenta un ejemplo de la aplicación del sistema PCV para el análisis de una interacción verbal entre un paciente hombre y un terapeuta hombre, tomada de un segmento correspondiente a la sesión 19 de una psicoterapia breve de modalidad individual y de orientación psicodinámica. En esta ilustración los turnos de palabra 29, 30, 31, 32 y 33 contienen la unidad de análisis del sistema PCV, es decir, las vocalizaciones de terapeuta y paciente. 
Como se aprecia en la Tabla 8, en el turno de habla 29 la alocución del terapeuta ha sido codificada con el PCV Conectado, pues esta impresiona al oyente como un habla elaborativa y orientada al paciente. A su vez, este turno de palabra, que corresponde a un único segmento de análisis, cumple con la condición de durar dos o más segundos, de manera que permite la percepción de los parámetros vocales principales para confirmar la codificación de dicho PCV. Del mismo modo, los turnos de habla 31 y 33 del terapeuta han sido codificados con el PCV Conectado.

En el turno de habla 30 la alocución del paciente ha sido codificada con dos PCV consecutivos: Afirmativo y Conectado. De esta manera, el turno de habla ha sido segmentado, pues el oyente aprecia de manera clara dos cualidades vocales diferentes en el habla del paciente. En el primer segmento el habla del paciente impresiona con convicción, mientras que en el segundo adquiere una sonoridad que impresiona a quien escucha por estar elaborada en el mismo momento de su emisión. A su vez, ambos segmentos cumplen con la condición de durar dos o más segundos, de manera que permite la percepción de los parámetros vocales principales para confirmar la codificación de los PCV ya descritos. Asimismo, el turno de habla 32, correspondiente al paciente, es considerado como un único segmento y codificado con el PCV Afirmativo.

Tabla 8

Ilustración de la Aplicación del Sistema de Codificación PCV

\begin{tabular}{lccc}
\hline \multicolumn{1}{c}{ Transcripción de turnos de habla } & $\begin{array}{c}\text { Unidad de análisis } \\
\text { (Segmento) }\end{array}$ & PCV \\
\hline 29. T: $\quad \begin{array}{l}\text { ¿Pero a qué le llamas desanimarte? ¿Qué te } \\
\text { hace sentir? }\end{array}$ & 1 & Conectado \\
30. P: $\quad \begin{array}{l}\text { Me siento frustrado, me siento así como } \\
\text { incapaz porque me pongo a pensar y cómo ellos } \\
\text { logran pensar a llegar a esa que hacen ellos }\end{array}$ & 1 & Afirmativo \\
30. P: $\quad$.. que tiene ellos o estudian mucho o no sé o en & 2 & Conectado \\
31. T: ¿Qué tiene ellos? & 1 & Conectado \\
32. P: $\quad$... ¿cómo que qué tienen? Yo creo que & 1 & Afirmativo \\
33. T: ¿Que qué tienen? & 1 & Conectado \\
\hline
\end{tabular}

Nota $. \mathrm{T}=$ terapeuta; $\mathrm{P}=$ paciente.

\section{Conclusiones}

El sistema de codificación de PCV, que demostró una alta confiabilidad, presenta al menos tres diferencias relevantes al elaborado por Rice y sus colaboradores (Rice \& Kerr, 1986; Rice \& Wagstaff, 1967; Wiseman \& Rice, 1989):

1. Describe PCV aplicables al habla hispana.

2. Propone un solo sistema de codificación aplicable tanto a paciente como a terapeuta. Esta característica del sistema permite hacer estudios comparativos y secuenciales de los PCV empleados por ambos a lo largo del proceso psicoterapéutico.

3. Describe tres categorías excluyentes de los PCV que permiten codificar los turnos vocales que no cumplen con las condiciones mínimas para determinar un PCV y que normalmente se presentan como fenómenos de conversación, tales como solapamientos y pausas llenas. 
4. Debido a que en la actualidad el estudio de la voz en psicoterapia ha vuelto a emerger como tópico de investigación de interés (Bucci, 2007; Campanelli, 2008, Junio; Campanelli et al., 2007; Russell, 1993; Tomicic et al., 2010), la importancia del sistema de codificación de PCV radica tanto en el carácter novedoso y poco explorado de su objeto de estudio como en sus posibles aplicaciones prácticas. Específicamente, constituye una herramienta que hará posible clarificar aspectos no verbales relacionados con la eficacia psicoterapéutica, los que contribuyen al proceso de cambio en los pacientes. Así, se puede pensar que en un futuro próximo los PCV puedan ser incorporados en el entrenamiento de psicoterapeutas de diferentes líneas teóricas.

Debido a la naturaleza del objeto y del procedimiento de análisis que propone el sistema de codificación de PCV, una limitación del sistema es que no ha sido validado con criterios externos. Por lo tanto, se hacen necesarios futuros estudios destinados a determinar la capacidad del sistema de discriminar PCV mediante una apreciación auditiva entrenada que efectivamente se asocie con mediciones objetivas relacionadas con los parámetros vocales empleados para caracterizarlos. Específicamente, se propone la realización de un estudio de validez concurrente por medio del análisis de segmentos codificados con cada PCV, utilizando como criterio externo parámetros acústicos objetivos de frecuencia fundamental, amplitud de la señal acústica y tasa discursiva a nivel suprasegmental (Bachowroski \& Owren, 1995, 2008, 2009), analizando la presencia diferencial de cada uno de estos parámetros en los distintos PCV.

No obstante la limitación recién mencionada, como producto de una línea de investigación más amplia de voz y psicoterapia (Tomicic et al., 2010), este sistema ya está siendo de utilidad para el análisis de prevalencia y secuencias temporales de PCV en episodios psicoterapéuticos relevantes, tales como episodios de cambio (Krause, 2005), de estancamiento (Herrera et al., 2009) y de ruptura de la alianza terapéutica (Martínez, Tomicic \& Medina, en prensa). Además, como resultado de estos análisis, el sistema de codificación de PCV permitirá responder preguntas relativas a las estrategias no verbales vocales de regulación mutua entre paciente y terapeuta en estos episodios relevantes (Tomicic, Martínez, et al., 2009), así como a las diferencias entre terapias exitosas y no exitosas.

\section{Referencias}

Álvarez-Gayou, J. L. (2004). Cómo hacer investigación cualitativa: fundamentos y metodología. México, DF, México: Paidós.

Andersen, P. A. (1999). Nonverbal communication: Forms and functions. Mountain View, CA: Mayfield.

Bachorowski, J.-A. \& Owren, M. J. (1995). Vocal expression of emotion: Acoustic properties of speech are associated with emotional intensity and context. Psychological Science, 6, 219-224. doi:10.1111/j.1467-9280.1995.tb00596.x

Bachorowski, J.-A. \& Owren, M. J. (2008). Vocal expressions of emotion. En M. Lewis, J. M. Haviland-Jones \& L. Feldman Barrett (Eds.), Handbook of emotions (3 ${ }^{\text {rd }}$ ed., pp. 196-210). New York, NY: The Guilford Press.

Bachorowski, J. \& Owren, M. J. (2009). Emotion in speech. En R. L. Squire (Ed.), Encyclopedia of neuroscience (pp. 897-901). Oxford, Reino Unido: Academic Press. doi:10.1016/B978-008045046-9.01897-0

Bady, S. (1985). The voice as a curative factor in psychotherapy. The Psychoanalytic Review, 72, 479-490.

Bakeman, R., McArthur, D., Quera, V. \& Robinson, B. F. (1997). Detecting sequential patterns and determining their reliability with fallible observers. Psychological Methods, 2, 357-370. doi:10.1037/1082-989X.2.4.357

Bakeman, R. \& Quera, V. (1995). Analyzing interaction: Sequential analysis with SDIS and GSEQ. New York, NY: Cambridge University Press.

Beebe, B., Jaffe, J., Lachmann, F., Feldstein, S., Crown, C. \& Jasnow, M. (2000). Systems models in development and psychoanalysis: The case of vocal rhythm coordination and attachment. Infant Mental Health Journal, 21, 99-122. doi:10.1002/(SICI)1097-0355(200001/04)21:1/2<99::AID-IMHJ11>3.0.CO;2-\#

Bucci, W. (2007). The role of bodily experience in emotional organization: New perspectives on the multiple code theory. En F. S. Anderson (Ed.), Bodies in treatment: The unspoken dimension (pp. 51-78). New York, NY: The Analytic Press.

Campanelli, L. (2008, Junio). Acoustic analysis of the voice: An implicit measure of emotional communication in the psychotherapeutic exchange. Ponencia presentada en la $39^{\text {th }}$ International Meeting of the Society for Psychotherapy Research, Barcelona, España.

Campanelli, L., Iberni, E., Sarracino, D., Degni, S. \& Mariani, R. (2007). Semiotics of the non verbal vocal expression of emotions and research into the psychotherapy process: A pilot study. Rivista di Psicologia Clinica, 1, $102-115$.

Campbell, N. (2007). On the use of non verbal speech sounds in human communication. En A. Esposito, M. FaundezZanuy, E. Keller \& M. Marinaro (Eds.), Verbal and nonverbal communication behaviours (pp. 117-128). Berlin, Alemania: Springer. 
Dodero, A., Hortas, M. A. S. \& Wilder, F. (2005). Metodología de estudio de alteraciones de la voz y baremos de incapacidad vocal. Cuadernos de Medicina Forense (Argentina), 1, 41-51.

Erickson, M. H. (1980). The nature of hypnosis and suggestion. En E. L. Rossi (Ed.), The collected papers of Milton H. Erickson on hypnosis (pp. 497-514). New York, NY: Irvington.

Herrera, P., Fernández, O., Krause, M., Vilches, O., Valdés, N. \& Dagnino, P. (2009). Revisión teórica y metodológica de las dificultades en psicoterapia: propuesta de un modelo ordenador. Terapia Psicológica, 27, 169-179. doi:10.4067/ S0718-48082009000200003

Holtz, P. J. (2004). The self- and interactive regulation and coordination of vocal rhythms, interpretive accuracy, and progress in brief psychodynamic psychotherapy. Dissertation Abstracts International, 64, 3526.

Knoblauch, S. H. (2000). The musical edge of therapeutic dialogue. Hillsdale, NJ: The Analytic Press.

Knoblauch, S. H. (2005). Body rhythms and the unconscious: Toward an expanding of clinical attention. Psychoanalytic Dialogues, 15, 807-827. doi:10.2513/s10481885pd1506_2

Krause, M. (2005). Psicoterapia y cambio: una mirada desde la subjetividad. Santiago, Chile: Ediciones Universidad Católica de Chile.

Krause, M., De la Parra, G., Arístegui, R., Dagnino, P., Tomicic, A., Valdés, N. ... Ben-Dov, P. (2007). The evolution of therapeutic change studied through generic change indicators. Psychotherapy Research, 17, 673-689. doi:10.108 0/10503300601158814

Krause, M., De la Parra, G., Arístegui, R. \& Strasser, K. (2006). Hacia una práctica efectiva en psicoterapia: estudio de episodios de cambio relevantes en diferentes tipos de psicoterapia y sus efectos en los resultados terapéuticos (Proyecto FONDECYT N ${ }^{\circ}$ 1030482). Manuscrito no publicado, Escuela de Psicología, Pontificia Universidad Católica de Chile, Santiago, Chile.

Krause, M., De la Parra, G., Arístegui, R. \& Strasser, K. (2008). Hacia una práctica efectiva en psicoterapia: estudio de episodios de cambio relevantes en diferentes tipos de psiocoterapia y sus efectos en los resultados terapéuticos (Informe Final Proyecto FONDECYT N 1030482). Manuscrito no publicado, Escuela de Psicología, Pontificia Universidad Católica de Chile, Santiago, Chile.

Landis, J. \& Koch, G. (1977). The measurement of observer agreement for categorical data. Biometrics, 33, $159-174$.

Le Huche, F. \& Allali, A. (1993). La voz: anatomía y fisiología de los órganos de la voz y del habla. Barcelona, España: Masson.

Martínez, C., Tomicic, A. \& Medina, L. (en prensa). Microanalysis of psychotherapeutic dialog: Dialogic discourse analysis in relevant psychotherapy episodes. International Journal for Dialogical Science.

McLeod, J. (2001). Qualitative research in counselling and psychotherapy. London, Reino Unido: Sage.

Moneta, M. E., Penna, M., Loyola, H., Buchheim, A. \& Káchele, H. (2008). Measuring emotion in the voice during psychotherapy interventions: A pilot study. Biological Research, 41, 389-395. doi:10.4067/S071697602008000400004

Osatuke, K., Humphreys, C. L., Glick, M. J., Graff-Reed, R. L., Mckenzie, L. T. \& Stiles, W. B. (2005). Vocal manifestations of internal multiplicity: Mary's voices. Psychology and Psychotherapy: Theory, Research and Practice, 78, 21-44. doi:10.1348/147608304X22364

Pell, M. D., Monetta, L., Paulmann, S. \& Kotz, S. A. (2009). Recognizing emotions in a foreign language. Journal of Nonverbal Behavior, 33, 107-120. doi:10.1007/s10919-008-0065-7

Ponterotto, J. G. (2006). Brief note on the origins, evolution, and meaning of the qualitative research concept "thick description". The Qualitative Report, 11, 538-549. Extraído de http://www.nova.edu/ssss/QR/QR11-3/ponterotto. pdf

Rice, L. N. \& Kerr, G. P. (1986). Measures of client and therapist vocal quality. En L. S. Greenberg \& W. M. Pinsof (Eds.), The psychotherapeutic process: A research handbook (pp. 73-106). New York, NY: The Guilford Press.

Rice, L. N. \& Wagstaff, A. K. (1967). Client voice quality and expressive style as indexes of productive psychotherapy. Journal of Consulting Psychology, 31, 557-563. doi:10.1037/h0025164

Rodríguez, G., Gil, J. \& García E. (1996). Metodología de la investigación cualitativa. Málaga, España: Ediciones Aljibe.

Russel, J. A., Bachorowski, J.-A. \& Fernandez-Dol, J. M. (2003). Facial and vocal expressions of emotion. Annual Review of Psychology, 54, 329-349. doi:10.1146/annurev.psych.54.101601.145102

Russell, R. (Ed.). (1993). Language in psychotherapy: Strategies of discovery. New York, NY: Plenum Press.

Scivetti, A. R. \& Garraza, A. M. (1996). El fenómeno vocal. San Luis, Argentina: Editorial Universitaria San Luis.

Scherer, K. R. (1982). Methods of research on vocal communication: Paradigms and parameters. En K. R. Scherer \& P. Ekman (Eds.), Handbook of methods in nonverbal behavior research (pp. 136-198). Cambridge, Reino Unido: Cambridge University Press.

Scherer, K. R. (1986). Vocal affect expression: A review and a model for future research. Psychological Bulletin, 99, 143-165. doi:10.1037/0033-2909.99.2.143

Scherer, K. R., Banse, R. \& Wallbott, H. G. (2001). Emotion inferences from vocal expression correlate across languages and cultures. Journal of Cross-Cultural Psychology, 32, 76-92. doi:10.1177/0022022101032001009

Scherer, K. R. \& Bergmann, G. (1990). Vocal communication. German Journal of Psychology, 8, 57-90.

Thompson, W. \& Balkwill, L.- L. (2006). Decoding speech prosody in five languages. Semiotica, 158, 407-424.

Tomicic, A., Chacón, D., Martínez, C., Guzmán, M., Bauer, S. \& Reinoso, A. (2009). Sistema de codificación de patrones de cualidad vocal de paciente y terapeuta (PCV-1.0): manual de entrenamiento y procedimiento para la codificación de patrones de cualidad vocal. Manuscrito no publicado.

Tomicic, A., Martínez, C., Altimir, C., Bauer, S. \& Reinoso, A. (2009). Coordinación vocal como una dimensión de la regulación mutua en psicoterapia. Revista Argentina de Clínica Psicológica, 18, 31-41. 
Tomicic, A., Martínez, C., Bauer, S., Reinoso, A., Guzmán, M. \& Chacón, L. D. (2010). El sonido de la psicoterapia: visión general de una línea de investigación. Gaceta de Psiquiatría Universitaria, 6, 98-107.

Wiseman, H. \& Rice, L.N. (1989). Sequential analyses of therapist-client interaction during change events: A taskfocused approach. Journal of Consulting and Clinical Psychology, 57, 281-286.

Fecha de recepción: Enero de 2010.

Fecha de aceptación: Octubre de 2010. 